\title{
Maximality of Reversible Gate Sets
}

\author{
Tim Boykett ${ }^{1,2,3(\varpi)} \mathbb{( 1 0}$ \\ 1 Institute for Algebra, Johannes-Kepler University, Linz, Austria \\ tim.boykett@jku.at, tim@timesup.org \\ 2 Time's Up Research, Linz, Austria \\ 3 University for Applied Arts, Vienna, Austria
}

\begin{abstract}
We investigate collections of reversible gates closed under parallel and serial composition. In order to better understand the structure of these collections of reversible gates, we investigate the lattice of closed sets and the maximal members of this lattice, that is, collections that are not all gates, but the addition of a single new gate will allow us to construct all gates. We find the maximal closed sets over a finite alphabet.
\end{abstract}

We then extend to ancilla and borrow closure for reversible gates. Here we find some structural results, including some examples.

Keywords: Reversible gates $\cdot$ Maximal closed classes $\cdot$ Permutation groups

\section{Introduction}

For a given finite set $A$, we investigate the collections of reversible gates, or bijections of $A^{k}$ for all $k$. The work derived from Tomasso Toffoli's work [14] and as such we call closed systems of bijections reversible Toffoli Algebras (RTAs). We also consider ancilla and borrow closure, where an extra input and output is allowed; an ancilla is provided and returned in a particular state, whereas a borrowed bit is provided and returned in an arbitrary state.

The work also relates to permutation group theory, as an RTA $C$ is a $\mathbb{N}$ indexed collection of permutations groups, $C^{[i]} \leq \operatorname{Sym}\left(A^{i}\right)$.

In previous papers, Aaronson, Grier and Schaeffer have determined all ancilla closed gates on a set of order 2 [1], and the author, together with Jarkko Kari and Ville Salo, has investigated generating sets [2,3] and other themes.

In this paper, we determine the possible maximal closed systems, relying strongly on Liebeck, Praeger and Saxl's work [11], and determine some properties of maximal borrow and ancilla closed RTAs.

We show that the maximal RTAs are defined by an index that defines the single arity at which the RTA is not the full set of bijections. We then show that for different indices and orders of $A$, only certain possibilities can arise. For

The research has been supported by Austrian Science Fund (FWF) research projects AR561 and P29931.

(C) Springer Nature Switzerland AG 2020

I. Lanese and M. Rawski (Eds.): RC 2020, LNCS 12227, pp. 206-217, 2020.

https://doi.org/10.1007/978-3-030-52482-1_12 
ancilla and borrow closed RTAs we find that there is similarly an index below which the maximal RTAs are full symmetry groups and above which they are never full.

We start by introducing the background properties of RTAs and some permutation group theory. The next section is an investigation of maximality, with the main result, Theorem 4, taking up the main body of this section. We then investigate properties of borrow and ancilla closed RTAs.

\section{Background}

In this section we will introduce the necessary terminology.

Let $A$ be a finite set. $\operatorname{Sym}(A)=S_{A}$ is the set of permutations or bijections of $A, A l t(A)$ the set of permutations of even parity. If $A=\{1, \ldots, n\}$ we will write $S_{n}$ and $A_{n}$. We write permutations in cycle notation and act from the right. We write the action of a permutation $g \in G \leq \operatorname{Sym}(A)$ on an element $a \in A$ as $a^{g}$. A subgroup $G \leq S_{A}$ is transitive if for all $a, b \in A$ there is a $g \in G$ such that $a^{g}=b$. We also say that $G$ acts transitively on $A$. If for all distinct $a_{1}, \ldots, a_{n} \in A$ and $b_{1}, \ldots, b_{n} \in A$ there is a $g \in G$ such that $a_{i}^{g}=b_{i}$ for all $i$, then we say $G$ is $n$-transitive on $A$. A subgroup $G$ of $S_{A}$ acts imprimitively if there is a nontrivial equivalence relation $\rho$ on $A$ such that for all $a, b \in A$, for all $g \in G, a \rho b \Rightarrow a^{g} \rho b^{g}$. If there is no such equivalence relation, then $G$ acts primitively on $A$.

Let $G$ be a group of permutations of a set $A$. Let $n \in \mathbb{N}$. Then the wreath product $G w r S_{n}$ is a group of permutations acting on $A^{n}$. The elements of $G w r S_{n}$ are $\left\{\left(g_{1}, \ldots, g_{n}, \alpha\right) \mid g_{i} \in G, \alpha \in S_{n}\right\}$ with action defined as follows: for $\left(a_{1}, \ldots, a_{n}\right) \in A^{n},\left(a_{1}, \ldots, a_{n}\right)^{\left(g_{1}, \ldots, g_{n}, \alpha\right)}=\left(a_{\alpha^{-1} 1}^{g_{1}}, \ldots, a_{\alpha^{-1} n}^{g_{n}}\right)$.

Let $B_{n}(A)=\operatorname{Sym}\left(A^{n}\right)$ and $B(A)=\bigcup_{n \in \mathbb{N}} B_{n}(A)$. We call $B_{n}(A)$ the set of $n$-ary reversible gates on $A, B(A)$ the set of reversible gates. For $\alpha \in S_{n}$, let $\pi_{\alpha} \in B_{n}(A)$ be defined by $\pi_{\alpha}\left(x_{1}, \ldots, x_{n}\right)=\left(x_{\alpha^{-1}(1)}, \ldots, x_{\alpha^{-1}(n)}\right)$. We call this a wire permutation. Let $\Pi=\left\{\pi_{\alpha} \mid \alpha \in S_{n}, n \in \mathbb{N}\right\}$. In the case that $\alpha$ is the identity, we write $i_{n}=\pi_{\alpha}$, the $n$-ary identity. Let $f \in B_{n}(A), g \in B_{m}(A)$. Define the parallel composition as $f \oplus g \in B_{n+m}(A)$ with $(f \oplus g)\left(x_{1}, \ldots, x_{n+m}\right)=$ $\left(f_{1}\left(x_{1}, \ldots, x_{n}\right), \ldots, \quad f_{n}\left(x_{1}, \ldots, x_{n}\right), \quad g_{1}\left(x_{n+1}, \ldots, x_{n+m}\right), \ldots, \quad g_{m}\left(x_{n+1}, \ldots\right.\right.$, $\left.x_{n+m}\right)$ ). For $f, g \in B_{n}(A)$ we can compose $f \bullet g$ in $\operatorname{Sym}\left(A^{n}\right)$. If they have distinct arities we "pad" them with identity, for instance $f \in B_{n}(A)$ and $g \in B_{m}(A)$, $n<m$, then define $f \bullet g=\left(f \oplus i_{m-n}\right) \bullet g$ and we can thus serially compose all elements of $B(A)$.

We call a subset $C \subseteq B(A)$ that includes $\Pi$ and is closed under $\oplus$ and $\bullet$ a reversible Toffoli algebra (RTA) based upon Toffoli's original work [14]. These have also been investigated as permutation clones [8], with ideas from category theory [9] and as memoryless computation [6]. If we do not insist upon the inclusion of $\Pi$, then we have reversible iterative algebras [3] in reference to Malcev and Post's iterative algebras. For a set $F \subseteq B(A)$ we write $\langle F\rangle$ as the smallest RTA that includes $F$, the RTA generated by $F$.

Let $C$ be an RTA. We write $C^{[n]}=C \cap B_{n}(A)$ for the elements of $C$ of arity $n$. We will occasionally write $\left(a_{1}, \ldots, a_{n}\right) \in A^{n}$ as $a_{1} a_{2} \ldots a_{n}$ for clarity. 
In any RTA $C$, the unary part $C^{[1]}$ is found as a wreath product in all other parts, $C^{[1]} w r S_{n} \leq C^{[n]}$ because the wire permutations give us the right hand factor while $f_{1} \oplus \cdots \oplus f_{n}$ for $f_{i} \in C^{[1]}$ gives us the left hand side.

Let $q$ be a prime power, $G F(q)$ the field of order $q, A G L_{n}(q)$ the collection of affine invertible maps of $G F(q)^{n}$ to itself. We note that for all $m \in \mathbb{N}$, $A G L_{n}\left(q^{m}\right) \leq A G L_{n m}(q)$. For a prime $p$, let $\operatorname{Aff}\left(p^{m}\right)=\bigcup_{n \in \mathbb{N}} A G L_{n m}(p)$ be the RTA of affine maps over $A=G F(p)^{m}$.

We say that an RTA $C \leq B(A)$ is borrow closed if for all $f \in B(A), f \oplus i_{1} \in C$ implies that $f \in C$. We say that an RTA $C \leq B(A)$ is ancilla closed if for all $f \in B_{n}(A), g \in C^{[n+1]}$ with some $a \in A$ such that for all $x_{1}, \ldots, x_{n} \in A$, for all $i \in\{1, \ldots, n\}, f_{i}\left(x_{1}, \ldots, x_{n}\right)=g_{i}\left(x_{1}, \ldots, x_{n}, a\right)$ and $g_{n+1}\left(x_{1}, \ldots, x_{n}, a\right)=a$ implies that $f \in C$. If an RTA is ancilla closed then it is borrow closed. For any prime power $q, \operatorname{Aff}(q)$ is borrow and ancilla closed.

\section{Maximality in Permutation Groups}

In this section we introduce some results from permutation group theory that will be of use. The maximal subgroups of permutation groups have been determined.

Theorem 1 ([11]). Let $n \in \mathbb{N}$. Then the maximal subgroups of $S_{n}$ are conjugate to one of the following $G$.

1. (alternating) $G=A_{n}$

2. (intransitive) $G=S_{k} \times S_{m}$ where $k+m=n$ and $k \neq m$

3. (imprimitive) $G=S_{m} w r S_{k}$ where $n=m k, m, k>1$

4. (affine) $G=A G L_{k}(p)$ where $n=p^{k}, p$ a prime

5. (diagonal) $G=T^{k}$.(Out $\left.(T) \times S_{k}\right)$ where $T$ is a nonabelian simple group, $k>1$ and $n=|T|^{(k-1)}$

6. (wreath) $G=S_{m}$ wr $S_{k}$ with $n=m^{k}, m \geq 5, k>1$

7. (almost simple) $T \triangleleft G \leq A u t(T), T \neq A_{n}$ a nonabelian simple group, $G$ acting primitively on $A$

Moreover, all subgroups of these types are maximal when they do not lie in $A_{n}$, except for a list of known exceptions.

It is worth noting that in the imprimitive case, $A$ is a disjoint sum of $k$ sets of order $m$, giving an equivalence relation with $k$ equivalence classes of order $m$, the wreath product acts by reordering the equivalence classes as $S_{k}$, then acting as $S_{m}$ on each equivalence class. In the wreath case, the set $A$ is a direct product of $k$ copies of a set of order $m$, the wreath product acts by permuting indices by $S_{k}$ then acting as $S_{m}$ on each index.

Lemma 1. Let $A$ be a set of even order and $n \geq 3$. Then $S_{A} w r S_{n} \leq \operatorname{Alt}\left(A^{n}\right)$.

Proof. $S_{A} w r S_{n}$ is generated by $S_{A}$ acting on the first coordinate of $A^{n}$ and $S_{n}$ acting on coordinates. 
The action of $S_{A}$ on $A^{n}$ is even because for each cycle in the first coordinate, the remaining $n-1$ coordinates are untouched. Every cycle occurs $|A|^{n-1}$ times, which is even, so the action of $S_{A}$ lies in $\operatorname{Alt}\left(A^{n}\right)$.

$S_{n}$ is generated by $S_{n-1}$ and the involution $(n-1 n)$. By the same argument, each cycle of the action occurs an even number of times, so the action of $S_{n-1}$ and the involution $((n-1) n)$ on $A^{n}$ lies in $A l t\left(A^{n}\right)$ so we are done.

We have a similar inclusion for affineness.

Lemma 2. For $n \geq 3, A G L_{n}(2) \leq A l t\left(2^{n}\right)$.

Proof. $A G L_{n}(2)$ is generated by the permutation matrices $\left\{\pi_{(1, i)} \mid i=2, \ldots, n\right\}$ and the matrix $\left[\begin{array}{ll}1 & 1 \\ 0 & 1\end{array}\right] \oplus i_{n-2}$. These bijections are even parity because they only act on two entries, thus have parity divisible by $2^{n-2}$ modulo 2 which is 0 .

Lemma 3. Let $A$ be even order. Then $S_{A} w r S_{2} \leq \operatorname{Alt}\left(A^{2}\right)$ iff 4 divides $|A|$.

Proof. The same argument as above applies for $S_{A}$. The action of $S_{2}$ swaps $\frac{|A|(|A|-1)}{2}$ pairs. This is even iff 4 divides $|A|$.

\section{Maximality in RTAs}

In this section, we will determine the maximal RTAs on a finite set $A$.

We have some generation results from other papers that will be useful.

Theorem 2 ([2] Theorem 5.9]). Let $A$ be odd. If $B_{1}(A), B_{2}(A) \subseteq C \subseteq B(A)$, then $C=B(A)$.

Theorem 3 ([3] Theorem 20]). If $A l t\left(A^{4}\right) \subseteq C \subseteq B(A)$ then $A l t\left(A^{k}\right) \subseteq C$ for all $k \geq 5$.

Lemma 4. Let $|A| \geq 3$, then $\left\langle B_{1}(A), B_{2}(A)\right\rangle$ is 3-transitive on $A^{3}$.

Proof. Let $A=\{1,2,3, \ldots\}$. Let $a, b, c \in A^{3}$ be distinct. We show that we can map these to $111,112,113 \in A^{3}$. There are three cases. See Fig. 1.

Case 1: Suppose $a_{3}, b_{3}, c_{3}$ all distinct. Let $\alpha=\left(\begin{array}{lll}a_{1} a_{3} & 1 a_{3}\end{array}\right)\left(b_{1} b_{3} \quad 1 b_{3}\right)$ $\left(c_{1} c_{3} 1 c_{3}\right) \in B_{2}(A)$. Let $\beta=\left(\begin{array}{lll}a_{2} a_{3} & 11\end{array}\right)\left(b_{2} b_{3} 12\right)\left(c_{2} c_{3} 13\right) \in B_{2}(A)$. Then $\gamma=\left(\pi_{(23)} \bullet\left(\alpha \oplus i_{1}\right) \bullet \pi_{(23)}\right) \bullet\left(i_{1} \oplus \beta\right)$ satisfies the requirements.

Case 2: Suppose $a_{3}, b_{3}, c_{3}$ contains two values, wlog suppose $a_{3}=b_{3}$. Let $d \in A-\left\{a_{3}, c_{3}\right\}$. Let $\delta=\left(a_{1} a_{3} a_{1} d\right) \in B_{2}(A)$. Let $\lambda=\pi_{(23)} \bullet\left(\delta \oplus i_{1}\right) \bullet \pi_{(23)}$. Then $\lambda$ will map $a, b, c$ to the situation in the first case.

Case 3: Suppose $a_{3}=b_{3}=c_{3}$. Then one of $a_{1}, b_{1}, c_{1}$ or $a_{2}, b_{2}, c_{2}$ must contain at least two values, wlog let $a_{1}, b_{1}, c_{1}$ be so. Then $\pi_{(13)}$ will give us the Case 1 if $a_{1}, b_{1}, c_{1}$ contains three values, Case 2 if $a_{1}, b_{1}, c_{1}$ contains two values.

The two following results are only relevant for even $A$. 

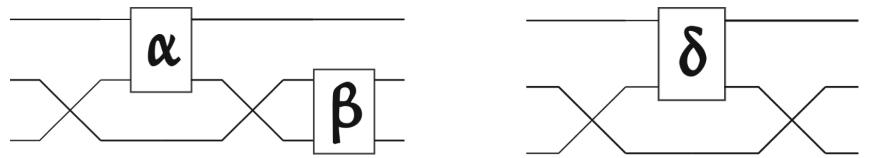

Fig. 1. Cases 1 and 2 in Lemma 4

Lemma 5. Let $|A| \geq 4, B_{1}(A), B_{2}(A) \subset C \leq B(A)$. Then $\operatorname{Alt}\left(A^{3}\right) \subseteq C^{[3]}$.

Proof. For $|A|=4$, the result is shown by calculation in $\operatorname{GAP}[7]$ that $\left\langle B_{1}(A) \oplus\right.$ $\left.i_{2} \cup B_{2}(A) \oplus i_{1} \cup \Pi^{[3]}\right\rangle$ as a subgroup of $B_{3}(A)$ is $\operatorname{Alt}\left(A^{3}\right)$.

For $|A|=5$ the result follows from Theorem 2 .

Suppose $|A| \geq 6$ Since $B_{2}(A) \subseteq C$, we have all 1-controlled permutations of $A$ in $C$. By [3] Lemma 18, with $P \subset A l t(A)$ the set of all 3 -cycles, we have all 2-controlled 3-cycles in $C$. Thus $(111112113) \in C . B_{1}(A) \cup B_{2}(A)$ is 3-transitive on $A^{3}$ by Lemma 4 , so we have all 3 -cycles in $C$, so $\operatorname{Alt}\left(A^{3}\right) \subseteq C$.

We know that this is not true for $A$ of order 2 , where $B_{2}(A)$ generates a group of order 1344 in $B_{3}(A)$, which is of index 15 in $A l t\left(A^{3}\right)$ and is included in no other subgroup of $B_{3}(A)$. However we find the following.

Lemma 6. Let $|A|$ be even, $B_{1}(A), B_{2}(A), B_{3}(A) \subset C \leq B(A)$. Then $\operatorname{Alt}\left(A^{4}\right) \subseteq$ $C^{[4]}$.

Proof. For $A$ of order 4 or more, we use the same techniques as in Lemma 5.

For $A$ of order 2, we calculate. We look at $C^{[4]}$ as a subgroup of $S_{16}$. The wire permutations $\Pi^{[4]}$ are generated by $(2,9,5,3)(4,10,13,7)(6,11)(8,12,14,15)$ and $(5,9)(6,10)(7,11)(8,12)$. Then $i_{1} \oplus B_{3}(A)$ is a subgroup of $B_{4}(A)$ acting on the indices $\{2,3,4\}$, generated by $(1,2,3,4,5,6,7,8)(9,10,11,12,13,14,15,16)$ and $(1,2)(9,10)$. It is a simple calculation to determine that this group is the entire alternating group $A_{16}$, so $\operatorname{Alt}\left(A^{4}\right) \subseteq C^{[4]}$.

We can now state our main theorem.

Theorem 4. Let $A$ be a finite set. Let $M$ be a maximal sub RTA of $B(A)$. Then $M^{[i]} \neq B_{i}(A)$ for exactly one $i$ and $M$ belongs to the following classes:

1. $i=1$ and $M^{[1]}$ is one of the classes in Theorem 1 .

2. $i=2,|A|=3$, and $M^{[2]}=A G L_{2}(3)$ (up to conjugacy)

3. $i=2,|A| \geq 5$ is odd and $M^{[2]}=S_{A} w r S_{2}$

4. $i=2,|A| \equiv 2 \bmod 4$ and $M^{[2]}=S_{A} w r S_{2}$

5. $i=2,|A| \equiv 0 \bmod 4$ and $M^{[2]}=\operatorname{Alt}\left(A^{2}\right)$

6. $i=2,|A| \equiv 0 \bmod 4$ and $M^{[2]}=T^{(3)} \cdot\left(\right.$ Out $\left.(T) \times S_{3}\right)$ where $T$ is a finite nonabelian simple group, with $|A|=|T|$ (up to conjugacy)

7. $i=2,|A| \equiv 0 \bmod 4$ and $M^{[2]}$ is an almost simple group (up to conjugacy)

8. $i \geq 3,|A|$ is even and $M^{[i]}=\operatorname{Alt}\left(A^{i}\right)$ 
Proof. Suppose $M<B(A)$ with $i \neq j$ natural numbers such that $M^{[i]} \neq B_{i}(A)$ and $M^{[j]} \neq B_{j}(A)$. Wlog, $i<j$, let $N=\left\langle M \cup B_{j}(A)\right\rangle$. Remember that compositions of mappings of arity at least $j$ will also be of arity at least $j$, so $N^{[k]}=M^{[k]}$ for all $k<j$. Then $M<N$ because $N$ contains all of $B_{j}(A)$ and $N<B(A)$ because $N^{[i]}=M^{[i]} \neq B_{i}(A)$. Thus $M$ was not maximal, proving our first claim.

For the rest of the proof, take $M$ maximal with $M^{[i]} \neq B_{i}(A)$. Then $M^{[i]}$ is a maximal subgroup of $B_{i}(A)$.

Suppose $i=1$. Then $B_{1}(A)=S_{A}$ and we are interested in the maximal subgroups of $S_{A}$. From Theorem 1 we know that these are in one of the 7 classes.

Suppose $i \geq 2$. Then $S_{A}^{i} \leq M^{[i]}$ so $M^{[i]}$ is transitive on $A^{i}$. As $\Pi^{[i]} \leq M^{[i]}$ we also know that $S_{A} w r S_{i} \leq M^{[i]}$. Assume $M^{[i]}$ acts imprimitively on $A^{i}$ with equivalence relation $\rho$. Let $a, b \in A^{i}, a \rho b$ with $a_{i} \neq b_{i}$. By the action of $S_{A}$ acting on the $i$ th coordinate we obtain $a^{\prime} \rho b^{\prime}$ with $a_{j}=a_{j}^{\prime}$ and $b_{j}=b_{j}^{\prime}$ for all $j \neq i$. By the action of $S_{i}$ on coordinates we can move this inequality to any index. Thus by transitivity we can show that $\rho=\left(A^{n}\right)^{2}$ and is thus trivial, so our action cannot be imprimitive.

We now consider the cases of $A$ odd and even separately.

Suppose $i \geq 2$ and $|A|$ is odd. If $i \geq 3$ then $M^{[1]}=B_{1}(A)$ and $M^{[2]}=B_{2}(A)$, so by Theorem 2 we have all of $B(A)$ and thus $M$ is not maximal, a contradiction. Thus we have $i=2$. $M^{[1]}=B_{1}(A)=S_{A}$ and $\pi_{(12)} \in M$ so $M$ contains $S_{A} w r S_{2}$. If $|A| \geq 5$ then by Theorem 1 this is maximal in $\operatorname{Sym}\left(A^{2}\right)$ so $M^{[2]}$ must be precisely this. So the case of $A$ order 3 is left. We want to know which maximal subgroups of $\operatorname{Sym}\left(A^{2}\right)$ contain $S_{A} w r S_{2}$. There are 7 classes of maximal subgroups, we deal with them in turn.

- Since $\pi_{(12)} \in M$ is odd on $A^{2}, M^{[2]} \nsubseteq \operatorname{Alt}\left(A^{2}\right)$.

- From the discussion above we know that $M^{[2]}$ is transitive and primitive on $A^{2}$, so the second and third cases do not apply.

- The permutations in $S_{3}$ can be written as affine maps in $\mathbb{Z}_{3}$ and $\pi_{(12)}$ can be written as $\left[\begin{array}{ll}0 & 1 \\ 1 & 0\end{array}\right]$, the off diagonal $2 \times 2$ matrix over $\mathbb{Z}_{3}$, so $S_{3} w r S_{2}$ embeds in the affine general linear group. Thus $M^{[2]}=A G L_{2}(3)$ is one possibility.

- The diagonal case requires $|T|^{k-1}=9$ for some nonabelian finite simple group $T$, a contradiction.

- The wreath case requires $9 \geq 5^{2}$, a contradiction.

- By [4] all $G$ acting primitively on $A^{2}$ with subgroups that are nonabelian finite simple groups are subgroups of $\operatorname{Alt}\left(A^{2}\right)$, and we have odd elements in $M$, so this is a contradiction.

Thus the only maximal subgroup is $M^{[2]}=A G L_{2}(3)$.

Suppose $i \geq 2$ and $|A|$ is even. We know from Theorem 3 that for $i>4$ we can get all of $\operatorname{Alt}\left(A^{i}\right)$ from $\cup_{1 \leq j<i} B_{j}(A)$. $\operatorname{Alt}\left(A^{i}\right)$ is maximal in $\operatorname{Sym}\left(A^{i}\right)$ so we are done.

Thus we are left with 3 cases, $i=2,3,4$.

From Lemma 6 we know that for $i=4, M^{[4]}=\operatorname{Alt}\left(A^{4}\right)$ is the only possibility. 
From Lemma 5 we know that for $i=3$ and $|A| \neq 2, M^{[3]}=\operatorname{Alt}\left(A^{3}\right)$ is the only possibility. For $|A|=2$ we find that $B_{2}(A)$ generates a subgroup of $B_{3}(A)$ that is only included in $\operatorname{Alt}\left(A^{3}\right)$, so again $M^{[3]}=\operatorname{Alt}\left(A^{3}\right)$ is the only possibility.

Thus we are left with the case $i=2$. From the above we know that the intransitive and imperfect cases cannot arise. Thus we need to consider the wreath, affine, diagonal and almost simple cases.

- $|A|=2: S_{A} w r S_{2}$ has order $8, B_{2}(2)$ has order 24 , so $M^{[2]}=S_{A} w r S_{2}$ is maximal and we are done.

- Case $6 \leq|A| \equiv 2 \bmod 4$ : Lemma 3 above says that $S_{A} w r S_{2} \not \leq A l t\left(A^{2}\right)$ so it is maximal by Theorem 1 .

- Case $|A|=4$ : Alternating is possible by inclusion. The affine case $A G L_{4}(2)$ lies in $A_{16}$ by Lemma 2. Diagonal not possible by order. Almost simple not possible because all primitive groups of degree 16 lie in the alternating group $A_{16}[4]$.

- Case $8 \leq|A| \equiv 0 \bmod 4$ : Alternating is always possible. If $A=2^{m}$ for some $m$, then $A G L_{m}(2)$ might be possible, but lies in $A l t\left(A^{2}\right)$ by Lemma 2 . Diagonal, almost simple might be possible, if $S_{A} w r S_{2} \leq M^{[2]}$.

\subsection{The Existence of Maximal RTAs}

It is not immediately clear that all the classes of maximal RTAs can actually exist. So let us investigate a few small examples.

Let us take $A$ of order 2. For $i=1$ we find no nontrivial subgroups, so the maximal is $M^{[1]}$ of order 1 . For $i=2$ case 4 gives us $S_{2} w r S_{2}$ of order 8 as a maximal subgroup. We note that $B_{2}(A)=A G L_{2}(2)$, i.e. all binary bijections are affine maps. For $i \geq 3$ we have $M^{[i]}$ alternating as the only example, as we know from Toffoli [14] and others that the alternating bijections of arity $i$ are generated by the collection of all permutations of arity less than $i$.

Taking $A$ of order 3 , we obtain a few more examples. For $i=1$ we write $A=$ $\{1,2,3\}$ and we know that $S_{3}$ has maximal subgroups $A_{3}$ as well as $\langle(12)\rangle,\langle(13)\rangle$, $\langle(23)\rangle$. These correspond in Theorem 1 to the alternating case and intransitive cases. For $i=2$ we write $A=Z_{3}$ and note that the unary maps are all affine, that is, the set of affine maps $\left\{x \mapsto a x+b \mid a, b \in \mathbb{Z}_{3}, a \neq 0\right\}$ is identical to the permutations $S_{3}=B_{1}(A)$. The binary affine maps $A G L_{2}(3)$ include all sums of unary affine maps and the wire permutation $\left[\begin{array}{ll}0 & 1 \\ 1 & 0\end{array}\right]$. With the inclusion of the linear map $(x, y) \mapsto(x+y, y)=\left[\begin{array}{ll}1 & 1 \\ 0 & 1\end{array}\right]$ we obtain all affine maps. From Theorem 1 above we know this is maximal as a subgroup of $B_{2}(A)$. For $i \geq 3$ we know that $B_{1}(A), B_{2}(A)$ generate all of $B(A)$ so we are done.

For $A$ of order 4 things get a touch more complex. For $i=1$ we get a number of maximal subgroups. $A_{4}$ is maximal. By fixing one element we obtain 4 maximal subgroups isomorphic to $S_{3}$ as intransitive subgroups. By imposing an 
equivalance relation with two classes of two elements each $(1,2 \mid 3,4$ or $1,3 \mid 2,4$ or $1,3 \mid 2,3)$ we obtain subgroups isomorphic to $S_{2} w r S_{2}$ that act imprimitively on $A$. $A G L_{2}(2)$ is of order 24 , same as $S_{4}$, we see that the affine maps are precisely the permutations, not maximal. There is no nonabelian simple group to allow a diagonal maximal subgroup. The wreath product also fails by order, and no nonabelian simple group of order less than 24 exists, so the almost simple case cannot arise. For $i \geq 2$ we find $M^{[2]}=A l t\left(A^{2}\right)$ a maximal subgroup. For $i=2$ we see that there are no nonabelian finite simple groups of order 16 , so case 6 cannot arise. It can be shown by investigation of [4] that $M^{[2]}$ cannot be an almost simple group.

For orders 5 and above, we know that the maximal RTAs for $i=1$ can be obtained by permutation group analysis directly. For $A$ of odd order we have the wreath case $S_{A} w r S_{2}$ maximal in $B_{2}(A)$ and none others. For $A$ even we have the alternating and wreath cases easily constructible. We are left with the question whether, for $A$ of order a multiple of 4 , the diagonal or almost simple cases can actually arise.

The possibilities for the diagonal case with $A$ of order equal to the order of a finite simple nonabelian group start with $A$ of order 60 . The other possibility is that $|A|^{2}=|T|$ for some finite simple nonabelian group $T$. The only known result in this direction is in [13] where they show that symplectic groups $S p(4, p)$ where $p$ is a certain type of prime, now known as NSW primes, have square order. The first of these groups is of order $\left(2^{4} \cdot 3 \cdot 5 \cdot 7^{2}\right)^{2}$ corresponding to $A$ of order $\left(2^{4} \cdot 3 \cdot 5 \cdot 7^{2}\right)=11760$. We note that the sporadic simple groups have order that always contains a prime to the power one, so they are not of square order. We know that the Alternating group can never have order that is a square, as the highest prime less than $n$ will occur exactly once in the order of the group. It might be possible that there are other finite simple groups of square order. As far as we are aware, there have been no further results in this direction.

Each of these possibilities is far beyond the expected useful arities for computational processes.

The other case is to look at almost simple groups. Let $A$ be of order $4 k$, then we are looking for an almost simple action of degree $16 k^{2}$. In [4] we saw that all primitive actions of degree 16 are alternating, that is, they are subgroups of $A_{16}$. In order to find an example, we can hope to use results about primitive permutation groups of prime power [5] and product of two prime power [10] degrees, so we would be able to investigate $A$ of order $4 k$ for $k \leq 14$. Once again this would include all examples of arities expected to be useful for computational processes.

\section{Maximality with Borrow and Ancilla Closure}

The strength of Theorem 4 is partially due to the fact that there is no effect of the existence of mappings of a certain arity in a given RTA on the size of the lower arity part, as there are no operators to lower the arity of a mapping. This does not apply with ancilla and borrow closure. In this section we collect some 
results about maximal ancilla and borrow closed RTAs. The following result reflects the first part of Theorem 4 .

Lemma 7. Let $M \leq B(A)$ be a maximal borrow or ancilla closed RTA. Then there exists some $k \in \mathbb{N}$ such that for all $i<k, M^{[i]}=B_{i}(A)$ and for all $i \geq k$, $M^{[i]} \neq B_{i}(A)$.

Proof. Suppose $M^{[k]}=B_{k}(A)$. Then for all $f \in B_{m}(A), m<k, f \oplus i_{k-m} \in M$ so by borrow closure $f \in M$, so $M^{[m]}=B_{m}(A)$ for all $m \leq k$. As $M$ is maximal, there must be a largest $k$ for which $M^{[k]}=B_{k}(A)$, since otherwise $M=B(A)$.

We will call $k$ the index of the maximal ancilla closed or borrow closed RTA. From Theorem 2 we then note the following.

Lemma 8. Let $|A|$ be odd. Then $M$ maximal with index $k=1,2$ are the only options.

In this case, we can say a bit more for index 2 . If $A$ is of order 3 , then by the argument in Theorem 4 above, we find that $M=\operatorname{Aff}(A)$, the affine maps over a field of order 3 . Otherwise $A$ is at least 5 and $B_{1}(A)$ is no longer affine. See Lemma 11 below.

Similarly, we obtain the following, but see Corollary 1 below for a stronger result.

Lemma 9. Let $|A| \geq 4$ be even. Then $M$ maximal with index $k=1,2,3$ are the only options and for $i>k, M^{[i]} \neq \operatorname{Alt}\left(A^{i}\right)$.

Proof. We start by noting that for even $|A|$, for all $f \in B_{i}(A), f \oplus i_{1} \in \operatorname{Alt}\left(A^{i+1}\right)$. Thus if $M^{[i]}=\operatorname{Alt}\left(A^{i}\right)$ for some $i>k$, then $M^{[i-1]}=B_{i-1}(A)$ which is a contradiction, which shows the second part of the result.

Suppose $k \geq 4$, so $B_{1}(A), B_{2}(A), B_{3}(A) \subseteq M$. Then by Lemma $6 \operatorname{Alt}\left(A^{4}\right) \subseteq$ $M$, so by Theorem $3 \operatorname{Alt}\left(A^{j}\right) \subseteq M$ for all $j \geq 5$. But we know that by borrow closure, this implies that $B_{j-1}(A) \subseteq M$ so $M$ is in fact $B(A)$. This is a contradiction, so $k<4$.

Using similar arguments, we obtain the following.

Lemma 10. Let $|A|=2$. Then $M$ maximal with index $k=1,2,3$ are the only options and for $i>k, M^{[i]} \neq \operatorname{Alt}\left(A^{i}\right)$.

Proof. Suppose $M$ is maximal with $k \geq 5$. Then by Theorem 3 we obtain $M^{[i]}=$ $\operatorname{Alt}\left(A^{i}\right)$ for all $i \geq 5$, which by the first argument in the previous Lemma, implies that $M$ is not maximal.

Suppose $M$ is maximal with $k=4$. We know that $M^{[3]}=B_{3}(A)$. Then by Lemma 6 we find that $M^{[4]}=\operatorname{Alt}\left(A^{4}\right)$, by Theorem 3 we obtain all of $\operatorname{Alt}\left(A^{5}\right)$ so by borrow closure all of $B_{4}(A)$ and thus $M$ is not maximal.

We obtain some examples of maximal borrow and ancilla closed RTA. The expression degenerate to describe maps where each output index depends only upon one input comes from [1]. 
Lemma 11. For $|A| \geq 5$, the degenerate RTA Deg $(A)$ generated by $B_{1}(A)$ is a maximal borrow closed RTA and maximal ancilla closed RTA.

Proof. Let $\operatorname{Deg}(A)$ be generated by $B_{1}(A)=S_{A}$. Then $\operatorname{Deg}(A)^{[i]}=S_{A} w r S_{i}$ for all $i \geq 2$ which is maximal in $B_{i}(A)$ by Theorem 1 . Thus any RTA $N$ properly containing $\operatorname{Deg}(A)$ will have $N^{[i]}=B_{i}(A)$ for some $i \geq 2$ and thus $N^{[2]}=B_{2}(A)$ by Lemma 7. Let $f \in N^{[2]}-\operatorname{Deg}(A)^{[2]}$, then $f \oplus f \in N[4]-\operatorname{Deg}(A)^{[4]}$ so $N^{[4]}=B_{4}(A)$ and by Lemmas 8 and $9, N=B(A)$, so $\operatorname{Deg}(A)$ is maximal.

For $|A|<5, B_{1}(A)$ consists of affine maps, so $\operatorname{Deg}(A)<\operatorname{Aff}(A)$ and thus cannot be maximal.

Corollary 1. Let $|A| \geq 4$ be even. Then $M$ maximal with index $k=1,2$ are the only options.

Proof. From Lemma 9 we know $k=1,2,3$ are possible. Suppose $M$ is maximal in $\mathrm{B}(\mathrm{A})$ with $k=3$.

Suppose $|A|=4 . B_{2}(A)$ can be embedded in $B_{4}(A)$ represented on $S_{256}$ with the tuples in $A^{4}$ represented by the integers $1, \ldots, 256$, generated by the permutations

\section{$(1,2,3,4,5,6,7,8,9,10,11,12,13,14,15,16)$}

$(17,18,19,20,21,22,23,24,25,26,27,28,29,30,31,32) \ldots$

... $(241,242,243,244,245,246,247,248,249,250,251,252,253,254,255,256)$

and $(1,2)(17,18) \ldots(241,242)$. With the wire permutations we obtain a subgroup of $S_{256}$ that is the alternating group, so $M^{[4]}=\operatorname{Alt}\left(A^{4}\right)$ and by Theorem 3 we then get $M^{[5]}=\operatorname{Alt}\left(A^{5}\right)$ and thus $M$ is not maximal.

Suppose $A$ is even with more than 6 elements. The degenerate RTA $\operatorname{Deg}(A) \leq$ $M$ because $M^{[1]}=B_{1}(A)$, but because $\operatorname{Deg}(A)$ is maximal and $M^{[2]}$ is a supergroup of $\operatorname{Deg}(A)^{[2]}, M$ is all of $B(A)$ and is not maximal.

Lemma 12. Let $A$ be of prime power order. Then $\operatorname{Aff}(A)$ is a maximal borrow closed RTA and a maximal ancilla closed RTA.

Proof. Let $M=\operatorname{Aff}(A)$. Suppose $M$ is not maximal, so $M<N<B(A)$.

Let $A$ be of odd order. For every $i$, except $i=1$ with $A$ of order $3, M^{[i]}$ is maximal in $B_{i}(A)$ by Theorem 1. Let $f \in B_{n}(A), f \in N-M$. Then $N^{[n]}=$ $B_{n}(A)$ by subgroup maximality, so for all $i<n, N^{[i]}=B_{i}(A)$. For all $j \in \mathbb{N}$, $f \oplus i_{j} \in(N-M)^{[n+j]}$ so similarly $N^{[n+j]}=B_{n+j}(A)$ so $N=B(A)$ and $M$ is maximal.

Let $A$ be of even order, so a power of 2. Let $f \in B_{n}(A), f \in N-M$. We know from Lemma 2 above that $M^{[n]} \leq \operatorname{Alt}\left(A^{n}\right)$ is not maximal, so the odd order argument above does not hold. By [12] we know that $N^{[n]}=B_{n}(A)$ or $N^{[n]}=\operatorname{Alt}\left(A^{n}\right)$. For all $j \in \mathbb{N}, f \oplus i_{j} \in(N-M)^{[n+j]}$ so $N^{[n+j]}=B_{n+j}(A)$ or $N^{[n+j]}=A l t\left(A^{n+j}\right)$. In both cases this means that $N^{[n+j-1]}=B_{n+j-1}(A)$, as for all $g \in B_{n+j-1}(A) g \oplus i_{1} \in \operatorname{Alt}\left(A^{n+j}\right) f$, so $N=B(A)$ and $M$ was maximal. 
Because $\operatorname{Aff}(A)$ is ancilla closed and maximal as borrow closed, there can be no ancilla closed RTA between $\operatorname{Aff}(A)$ and $B(A)$ so $\operatorname{Aff}(A)$ is a maximal ancilla closed RTA.

We look at a few concrete examples.

By [1] we know that for $A$ of order 2, we have the following maximal ancilla closed RTAs.

- The affine mappings,

- The parity respecting mappings, which either preserve the number of 1 s mod 2 , or invert it,

- The odd prime-conservative mappings, that preserve the number of $1 \mathrm{~s}$ mod $p$, an odd prime.

The affine mappings have index 3, the parity respecting index 2 and the odd prime-conservative mappings have index 1 .

It remains an open problem whether these are the borrow closed maximal RTAs over $A$ of order 2 .

For $A$ of order 3 , we know that the affine maps Aff(3) is an index 2 maximal borrow closed RTA and a maximal ancilla closed RTA.

For $A$ of order 4 , we can say the following about index 2 maximals. There are the following inclusions, $S_{4} w r S_{2}<A S p<A G L_{4}(2)<A l t\left(4^{2}\right)$ where $A S p$ is a group of order 11520 that consists of the affine maps where the linear part is a symplectic linear map in $S p(4,2)$. If $M^{[2]}=\operatorname{Alt}\left(4^{2}\right)$ then $M$ includes the affine maps properly. We know that the affine maps are maximal, a contradiction. $M^{[2]}=A G L_{4}(2)$ for the affine maps that we know form a maximal borrow and ancilla closed RTA. It is possible that $M^{[2]}=S_{4} w r S_{2}$ or $M^{[2]}=A S p$ for some maximal $M$.

For $A$ of order 5 or more, we know that index 2 arises only for the degenerate RTA $\operatorname{Deg}(A)$.

\section{Conclusion and Further Work}

We have determined the maximal RTAs, using results from permutation group theory and some generation results.

As we have not been able to construct explicitly an example of a maximal RTA with $i=2$ and $M^{[i]}$ of diagonal or almost simple type, the conjecture remains that these are not, in fact, possible. We note however that if such examples exist, they will arise for $A$ of order 8 or more, so will probably not be relevant for any practical reversible computation implementation.

In future work we aim to determine the weight functions as described by [8] for maximal RTAs, in order to determine whether they hold some interesting insights.

The results for borrow and ancilla closed RTAs are not as comprehensive. We hope to determine these in the foreseeable future. We note interestingly that for a state set of order 5 or more, Lemma 11 indicates that if we can implement 
all permutations of the state set, we need only have one non-degenerate gate in order to implement all gates under borrow or ancilla closure. Similarly we see that once we can implement all affine maps on a state set of prime power order, then only one nonaffine gate is needed to implement all gates. For the ancilla case, many of the techniques of [1] will prove useful. In the ancilla case, we know all maximal RTA with index 2 except for $A$ of order 4 .

Acknowledgements. Michael Guidici has helped extensively with understanding primitive permutations groups, for which I thank him greatly.

\section{References}

1. Aaronson, S., Grier, D., Schaeffer, L.: The classification of reversible bit operations. Electron. Colloquium Comput. Complexity (66) (2015). https://eccc.weizmann.ac. il//report/2015/066/

2. Boykett, T.: Closed systems of invertible maps. J. Multiple-Valued Logic Soft Comput. 32(5-6), 565-605 (2019)

3. Boykett, T., Kari, J., Salo, V.: Finite generating sets for reversible gate sets under general conservation laws. Theor. Comput. Sci. 701(C), 27-39 (2017). https://doi. org $/ 10.1016 /$ j.tcs.2016.12.032

4. Buekenhout, F., Leemans, D.: On the list of finite primitive permutation groups of degree $\leq$ 50. J. Symb. Comput. 22(2), 215-225 (1996). https://doi.org/10.1006/ jsco.1996.0049

5. Cai, Q., Zhang, H.: A note on primitive permutation groups of prime power degree. J. Discrete Math. 2, 191-192 (2015)

6. Gadouleau, M., Riis, S.: Memoryless computation: new results, constructions, and extensions. Theoret. Comput. Sci. 562, 129-145 (2015). https://doi.org/10.1016/ j.tcs.2014.09.040

7. The GAP Group: GAP - Groups, Algorithms, and Programming, Version 4.10.2 (2019). https://www.gap-system.org

8. Jeřábek, E.: Galois connection for multiple-output operations. Algebra Universalis 79(2), 1-37 (2018). https://doi.org/10.1007/s00012-018-0499-7

9. LaFont, Y.: Towards an algebraic theory of Boolean circuits. J. Pure Appl. Algebra 184, 257-310 (2003)

10. Li, C.H., Li, X.: On permutation groups of degree a product of two prime-powers. Commun. Algebra 42(11), 4722-4743 (2014). https://doi.org/10.1080/00927872. 2013.823500

11. Liebeck, M.W., Praeger, C.E., Saxl, J.: A classification of the maximal subgroups of the finite alternating and symmetric groups. J. Algebra 111(2), 365-383 (1987). https://doi.org/10.1016/0021-8693(87)90223-7

12. Mortimer, B.: Permutation groups containing affine groups of the same degree. J. Lond. Math. Soc. 2(3), 445-455 (1977). https://doi.org/10.1112/jlms/s2-15.3.445

13. Newman, M., Shanks, D., Williams, H.C.: Simple groups of square order and an interesting sequence of primes. Acta Arith. 38(2), 129-140 (1980)

14. Toffoli, T.: Reversible computing. In: de Bakker, J., van Leeuwen, J. (eds.) ICALP 1980. LNCS, vol. 85, pp. 632-644. Springer, Heidelberg (1980). https://doi.org/10. 1007/3-540-10003-2_104 